\title{
Restauración de montes quemados en condiciones mediterráneas
}

Recibido: 22 xaneiro 2009 / Aceptado: 3 abril 2009

(C) IBADER- Universidade de Santiago de Compostela 2009

\begin{abstract}
Wildfires may produce ecosystem damages that would require post-fire mitigation and/or restoration actions. The question is what are the criteria to identify those burned areas that show high degradation risk in order to plan and prioritise restoration projects. To address that question it is necessary to start with the analysis of fire impact, and from that analysis to derive predictive tools for assessing the fragility and regeneration capacity of burned ecosystems. The identification of post-fire degradation mechanisms provides the basis for developing the corresponding specific mitigation/restoration actions. The diagnostic of ecological impact of wildfires together with the established forest management objectives allow deriving mitigation/restoration strategies and the subsequent implementation projects.

We present our experience on the evaluation of post-fire ecosystem vulnerability and on the assessment of restoration planning derived from recent and ongoing EC research projects. This includes the development of shortterm restoration techniques suited for degraded soils and dry Mediterranean conditions, where fire-induced degradation is complicated with water shortage for regenerating vegetation.
\end{abstract}

Key words Post-fire restoration · Vulnerability · Soil erosion - Emergency seeding

Victoriano Ramón Vallejo

Fundacion Centro Estudios Ambientales del Mediterraneo, www.gva.es/ceam

email: ramonv@ceam.es

Susana Bautista

Departamento Ecología, Universidad de Alicante

José Antonio Alloza

Fundacion Centro Estudios Ambientales del Mediterraneo, www.gva.es/ceam

\section{Introducción}

La restauración ecológica forestal tiene como objetivo intrínseco la recuperación de montes degradados y de su sostenibilidad, $y$, por lo tanto, la lucha contra la desertificación. Además de la propia recuperación del ecosistema, se pretende disminuir el riesgo de catástrofes naturales asociadas directa o indirectamente a la degradación del monte, como las inundaciones, la pérdida de suelo y su acumulación aguas abajo con efectos negativos en infraestructuras o la movilidad de las dunas en zonas habitadas. En tiempos recientes ha tomado gran relevancia el fenómeno de los grandes incendios forestales que en ocasiones genera la necesidad de la restauración después del fuego y también la necesidad de la gestión del monte para la prevención de incendios. Todas estas catástrofes naturales, facilitadas por la degradación del territorio, han causado y siguen causando pérdidas de vidas humanas y daños económicos. En el ámbito Mediterráneo, las situaciones críticas corresponden a la transición entre clima seco sub-húmedo y semiárido, donde confluye un alto riesgo de perturbaciones (como el fuego) y un elevado estrés hídrico. Por lo tanto, los esfuerzos de restauración en la lucha contra la desertificación deben priorizarse en estas regiones.

\section{La restauración de zonas quemadas y su integración en la planificación forestal}

La metodología integra los conocimientos y experiencias adquiridas durante más de 15 años de investigación en ecosistemas mediterráneos, con la información cartográfica disponible. En síntesis, se analizan factores asociados a la vegetación, concretamente el potencial de regeneración evaluado en base a la información contenida en la versión digital del Mapa Forestal (Ruiz de la Torre, 1990), con los factores del medio que condicionan esta potencialidad. 


\section{Factores asociados a la vegetación}

\section{Potencial de autosucesión}

En los ecosistemas mediterráneos, como criterio general y en términos de potencialidad, después del fuego tiene lugar un proceso de autosucesión: las comunidades vegetales incendiadas se reinstalan y vuelven a conformar las mismas comunidades a corto plazo (Trabaud, 1994, 1999, 2000). Esta dinámica se cumple en las comunidades dominadas por pino carrasco y pinaster (Ne'eman \& Trabaud, 2000; Kazanis \& Arianoutsou, 2002; Gallegos et al., 2003), aunque en estos casos esté en función del régimen de incendios, régimen climático o capacidad reproductiva de los pinos (Ne'eman et al, 1999; Pausas, 2001).

En la metodología utilizada se asume el proceso de autosucesión excepto en las teselas cuya única especie arbórea está constituida por pinos; para determinar la capacidad reproductiva de estas masas de pinar, se ha considerado la especie de pino y su edad. El Mapa Forestal únicamente contiene información sobre los intervalos de altura de las masas, sin indicar su edad, motivo por el cual se ha tenido que recurrir a curvas de calidad que relacionan altura y edad para cada especie de pino (Gandullo \& Sánchez Palomares, 1994). En su valoración, se han considerado tres categorías cualitativas:

Buena: Criterio general para todas las teselas, excepto aquellas que tienen únicamente pinos como especies arbóreas. En este último caso, se ha considerado con buena capacidad de autosucesión a las teselas que están ocupadas exclusivamente por pino carrasco o pinaster (como especie arbórea) y con una altura superior a los $3 \mathrm{~m}$. Dentro de los intervalos de altura utilizados por el Mapa Forestal, el límite de los $3 \mathrm{~m}$ garantiza la edad reproductiva del arbolado.

Media: Teselas cuya única especie arbórea es pino carrasco o pinaster y presentan una altura comprendida entre 1.5 y $3 \mathrm{~m}$ (intervalo de altura establecido por el Mapa Forestal). A diferencia de la categoría anterior, para este intervalo de altura no hay plena seguridad de que el arbolado haya alcanzado la madurez reproductiva.

Baja: Resto de casos en los cuales la única especie arbórea es el pino; se corresponden básicamente con teselas de pino carrasco o pinaster muy jóvenes o teselas ocupadas por pino laricio, especie cuya regeneración después del fuego es problemática (Rodrigo et al., 1999).

Zonas degradadas: No se consideran las áreas catalogadas como degradadas (desierto o semidesierto en la terminología del Mapa Forestal). Si bien la carga de combustible es muy baja (y por tanto el riesgo de incendios también), su escasa cobertura las hace, en términos generales, muy vulnerables.

No valorable: Teselas en las que no se ha podido realizar la valoración. Corresponden básicamente a parte de la superficie ocupada por mosaico agrícola-forestal o áreas en proceso de urbanización, para las cuales no hay información acerca de la altura o composición del arbolado.

\section{Velocidad de regeneración}

La distinta estrategia reproductiva (germinadora o rebrotadora) de las especies vegetales, origina importantes diferencias en la rapidez de respuesta (regeneración) inmediatamente después del fuego. Se ha evaluado la velocidad teórica de regeneración de cada tesela en función de las especies presentes; para ello, se ha asignado la estrategia reproductiva a cada una de las cuatro posibles especies (o formaciones vegetales) principales. Para cada tesela, la valoración se ha establecido atendiendo al porcentaje de superficie ocupada por especies rebrotadoras, ya que éstas presentan un rápido recubrimiento del suelo poco después del incendio (Ferran et al. 1992; Abad et al, 1997; Vallejo \& Alloza, 1998; Pausas \& Vallejo, 1999). Las categorías establecidas han sido:

Alta: Presencia de especies rebrotadoras en un mínimo del $40 \%$ de la superficie de la tesela. Se considera que la protección del suelo por la vegetación es efectiva a partir del $30-40 \%$ de recubrimiento (Thornes, 1995); por tanto, con este requisito, se asegura una rápida protección frente a la erosión con una rápida y eficaz respuesta de la vegetación (presencia de rebrotadoras y umbral mínimo de recubrimiento del suelo respectivamente).

Media: Presencia de rebrotadoras con cobertura inferior al $40 \%$ o no determinada, o especies con estrategia mixta (rebrotadora/germinadora).

Baja: Presencia exclusiva de especies germinadoras.

Zonas degradadas: Dada su vulnerabilidad intrínseca, se ha mantenido como categoría diferenciada.

No valorable: Teselas en las cuales la falta de información en el Mapa Forestal impide la valoración.

\section{Capacidad potencial de regeneración}

El potencial de autosucesión y la velocidad de regeneración se han integrado en una sola variable que resume la capacidad potencial de regeneración. La integración se ha realizado por medio de una valoración cualitativa (Tabla 1), aplicando un método matricial (MMA, 2000).

\section{Factores del medio físico}

La degradación del suelo queda definida como la disminución en la capacidad, actual o potencial, del suelo para producir (cuantitativa y cualitativamente) bienes y servicios (FAO, 1979). Actualmente existen varias metodologías que evalúan la degradación del suelo y la desertificación (FA0, 197

9; Kosmas, 1999; Sánchez Díaz, 2001). Sin embargo, no existen criterios que aporten resultados satisfactorios y globalmente aceptados, entre otras causas, por la escasa información disponible (Sánchez Díaz, 2001). 


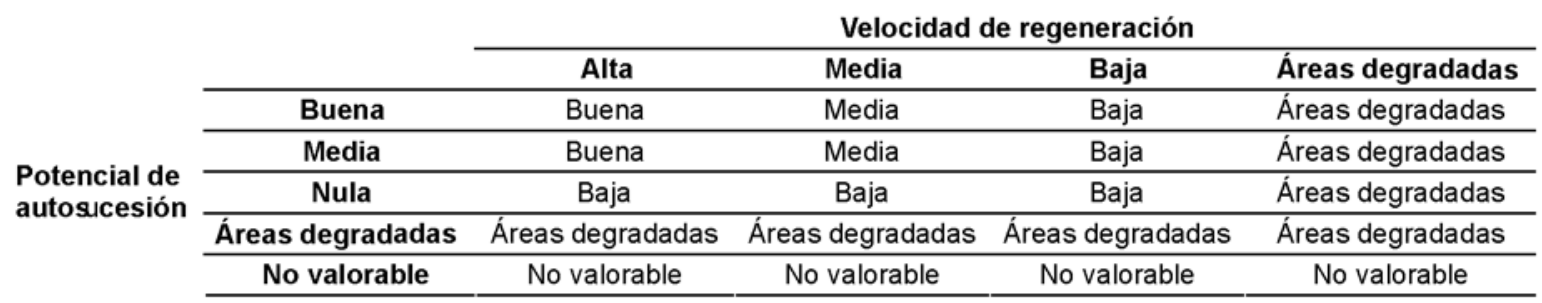

Tabla 1.- Integración de la información del potencial de autosucesión y la velocidad de regeneración, para obtener la capacidad potencial de regeneración

Partiendo de la complejidad del fenómeno y las limitaciones cartográficas, en este estudio se ha realizado una aproximación al riesgo de degradación física a partir de dos variables: riesgo de erosión e intensidad del período seco, aspectos ambos de gran incidencia en los procesos de regeneración de la vegetación después de un incendio.

\section{Estados erosivos}

El control de la erosión ha sido uno de los principales objetivos en la planificación de las actuaciones de repoblación y uno de los criterios determinantes para seleccionar zonas de actuación; por ejemplo, el Plan Nacional de Acciones Prioritarias en Materia de Restauración Hidrológico-Forestal o el Programa de Restauración Hidrológico Forestal y Control de la Erosión. En estos planes o programas, la determinación del riesgo de erosión se realiza a partir de los criterios de la Ecuación Universal de Pérdidas de Suelo (USLE).

La USLE es el modelo de predicción de pérdidas de suelo con mayor aceptación y de más amplia utilización, ya que busca una máxima facilidad de aplicación (Castillo \& Albadalejo, 1992). Sin embargo, este modelo no abarca todos los factores relevantes del fenómeno, el volumen de escorrentía no está explícitamente considerado, se establecen relaciones lineales entre todos los factores y no tiene en cuenta algunas formas de erosión (Castillo \& Albadalejo, 1992), originando importantes sobrevaloraciones en las estimaciones de pérdidas de suelo (Esteve et al, 1990; Sánchez, 1997; Boix-Fayos et al, 2003).

Para corregir las sobrevaloraciones aportadas por la aplicación de la USLE, se ha procedido a efectuar un ajuste atendiendo a la litología. La cobertura de erosión potencial (Antolín, 1998) se ha cruzado con la de litologías (COPUT, 1998) para reducir al nivel más bajo de erosión potencial todas las teselas situadas sobre afloramientos de calizas o dolomías, ya que estas litologías desarrollan suelos muy permeables (Lepart \& Debussche, 1992) y poco erosionables (Cerdá, 1999).

\section{Periodo seco}

Como estimador de la intensidad del periodo seco se ha calculado la Intensidad Bioclimática Seca (IBS). En su cálculo se ha utilizado la metodología de Montero de Burgos \& González Rebollar (1983) con las siguientes consideraciones:

- En correspondencia con la escasa profundidad de los suelos forestales valencianos, en el cálculo de los balances se ha estimado una reserva de $50 \mathrm{~mm}$ para todo el territorio forestal.

- Para estimar las pérdidas por escorrentía se ha considerado el relieve de cada tesela forestal. Para ello, se ha superpuesto la cobertura de unidades de paisaje (tomada de Antolín, 1998), asumiendo un porcentaje de escorrentía del $10 \%$ en las teselas situadas en una pendiente inferior al $8 \%$ y del $30 \%$ en el resto de situaciones.

- En el balance hídrico, realizado para 110 estaciones termopluviométricas (datos tomados de Pérez Cueva, 1994), se ha calculado la evapotranspiración potencial según el modelo de Thornthwaite.

Los resultados de IBS se han extrapolado al territorio aplicando un krigeado.

\section{Riesgo de degradación}

El riesgo de erosión e intensidad del periodo seco se han integrado, utilizando una valoración cualitativa, en una variable denominada Riesgo de degradación debido a factores físicos. Las categorías consideradas se indican en la Tabla 2.

\begin{tabular}{lllll} 
& & \multicolumn{3}{c}{ Intensidad bioclimática seca } \\
\cline { 2 - 5 } Erosión potencial & & Alta & Media & Baja \\
\hline & Baja & Alto & Medio & Bajo \\
\cline { 2 - 5 } & Media & Alto & Medio & Medio \\
\cline { 2 - 5 } & Alta & Muy alto & Alto & Medio \\
\hline
\end{tabular}

Tabla 2.- Integración de la información para obtener el riesgo de degradación, en función de las categorías de erosión potencial e intensidad bioclimática seca 


\section{Análisis del impacto de la extracción o no de la madera quemada}

El arrastre de troncos realizados durante los dos primeros años después del fuego produce erosión lineal (regueros) significativa a partir de los arrastraderos y vías de saca en vertientes con substrato deleznable, de tipo margas, arcillas y areniscas (Bautista et al., 2004). No hay efectos erosivos significativos sobre substratos consolidados de tipo calizas. La vegetación, y el regenerado de pinar, no se ven afectados de forma significativa por los tratamientos. De estos resultados se deduce la recomendación de extremar las precauciones en la extracción de troncos quemados en suelos frágiles, realizándola mediante técnicas de cableado que eviten los arrastres, o bien retrasando la extracción hasta que el regenerado proteja el suelo y evitando que los arrastres produzcan incisiones lineales.

Una de las razones que se consideran a la hora de defender la extracción de la madera quemada es la colonización y proliferación de escolítidos (géneros Ips y Tomicus, Coleoptera: Scolytidae) en los troncos quemados, que pueden actuar así de foco de infección para las masas de pinar cercanas

La susceptibilidad de los árboles a la infección por escolítidos aumenta con la aparición de algún tipo de daño mecánico o debilitamiento producido por fuego, estrés hídrico, nevadas, etc. El conjunto de resultados experimentales y observaciones disponibles para este tipo de insectos perforadores parecen sugerir que poseen cierta capacidad para colonizar la madera quemada.

En la prospección de pinares de Pinus halepensis quemados con diferente intensidad, en los que se había producido un foco de infección por Tomicus destruens, se observó que la mayor proporción de árboles colonizados por el perforador se produjo en el conjunto de individuos parcialmente quemados, en los que las acículas -total o parcialmente secas- se conservaban en las copas. No se observaron entradas de escolítidos en árboles completamente quemados con hojas consumidas. A partir de estos resultados, se planteó la hipótesis de que el nivel de daño producido por el fuego afectaría la composición terpénica de los pies de pino y, como consecuencia, la respuesta de atracción primaria de $T$. destruens.

Se ha podido constatar que niveles de daño producidos por el fuego afectan a la composición terpénica de la oleorresina de los pinos $\mathrm{y}$, como consecuencia, a la respuesta de atracción primaria de Tomicus destruens. La producción de terpenos en los árboles quemados fue mayor en los pies afectados de forma menos severa y se redujo gradualmente en los casos de severidad media y alta. Los resultados obtenidos en los ensayos de campo con trampas cebadas con a-pineno (componente mayoritario de las oleorresinas de pino carrasco), mostraron una respuesta positiva de Tomicus destruens a este compuesto. Los terpenos producidos en los árboles quemados son candidatos potenciales para actuar como señales de atracción primaria para escolítidos. El que la mayor producción de una gran variedad de terpenos se produzca en los árboles menos afectados, señala a este tipo de pies como los elementos de mayor riesgo en un área quemada. En conjunto, los resultados de la prospección de campo y de otros trabajos previos revelan una preferencia de las especies del género Tomicus más comunes en nuestra zona por los árboles en los que no se haya producido una combustión severa o la muerte inmediata tras el incendio.

La eficacia de la extracción de la madera como medida preventiva contra las plagas de escolítidos se limita a los 69 primeros meses tras el incendio. No obstante, debe tenerse en cuenta que este periodo es el de máximo riesgo de degradación y erosión del suelo por lo que deben sopesarse adecuadamente las condiciones ambientales y las prioridades en cada zona. Se recomiendan actuaciones selectivas, que concedan prioridad a los árboles parcialmente afectados por el fuego en caso de riesgo de plaga de escolítidos, que eviten las áreas más sensibles y de mayor riesgo de erosión, que apliquen medidas correctoras en los casos de riesgo y que se ejecuten de forma heterogénea, dejando un mosaico de rodales con y sin actuación para mantener el papel estructural y funcional de ramas y troncos.

\section{Las técnicas de restauración}

Las siembras de emergencia consisten en la utilización de especies herbáceas autóctonas de rápida respuesta, acompañas de la aportación superficial de material orgánico de partícula gruesa en forma de acolchado (mulching), utilizando paja, residuos de podas y desbroces forestales $u$ otros similares. Estos tratamientos han demostrado ser eficientes en aumentar la cubierta vegetal a corto plazo y disminuir las tasas de erosión y escorrentía (Bautista et al. 1994).

En las plantaciones, se han ensayado una gran diversidad de especies leñosas mediterráneas, tanto arbustivas como

Capacidad de regeneración

\begin{tabular}{lllcccc}
\cline { 2 - 7 } & & Baja & Media & Alta & Áreas degradadas & No valorable \\
\hline \multirow{2}{*}{$\begin{array}{c}\text { Riesgo de } \\
\text { degradación }\end{array}$} & Muy alto & Muy alta & Muy alta & Alta & Muy alta & No valorable \\
\cline { 2 - 7 } & Alto & Muy alta & Alta & Media & Muy alta & No valorable \\
\cline { 2 - 7 } & Medio & Alta & Media & Baja & Alta & No valorable \\
\cline { 2 - 7 } & Bajo & Media & Baja & Baja & Media & No valorable \\
\hline
\end{tabular}

Tabla 3.- Integración de las categorías de capacidad de regeneración y riesgo de erosión, para evaluar la fragilidad de la vegetación frente a incendios forestales 


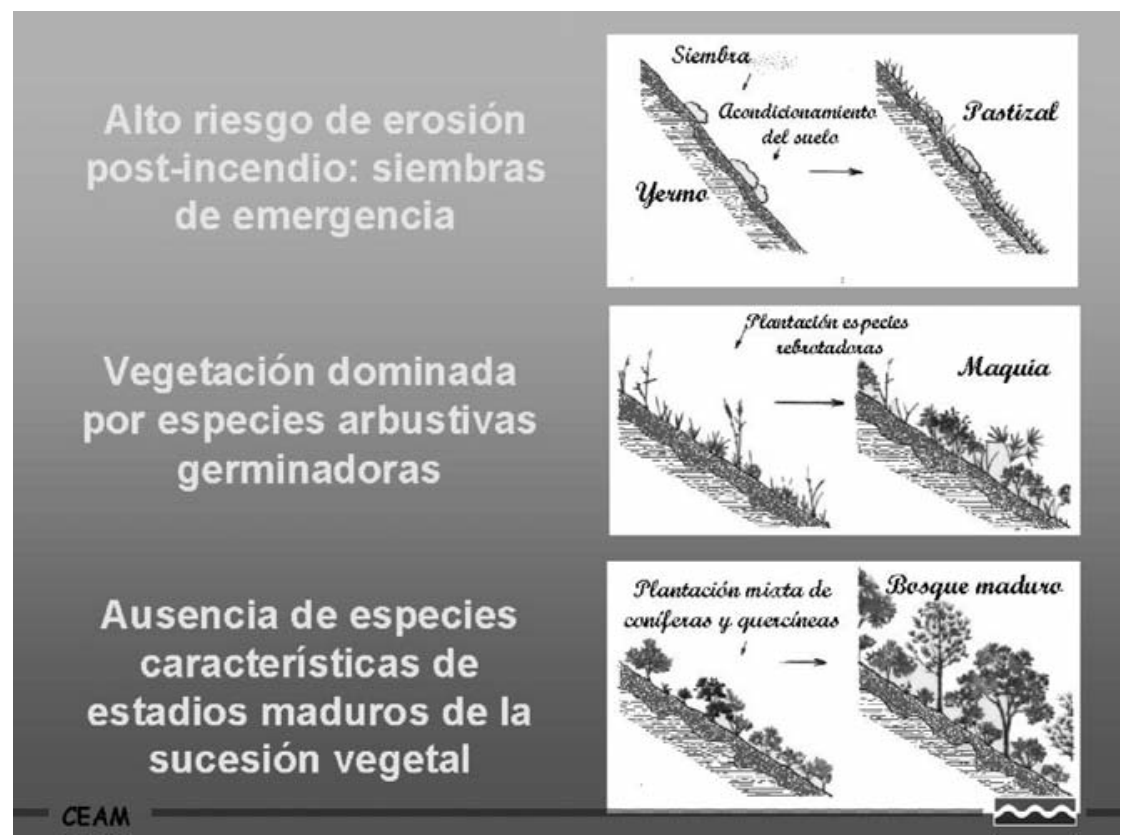

Figura 1.- Estrategias de restauración forestal según el estado de degradación del ecosistema arbóreas. La mortalidad de los plantones generalmente se produce durante el primer verano en el campo, cuando el plantón todavía no ha arraigado en el suelo y puede sufrir una fuerte sequía. La salida de las raíces del plantón del cepellón y la colonización rápida y profunda del suelo son factores críticos en la supervivencia. Las causas primarias son la sequía, agravada por la poca capacidad de retención de agua en el caso de suelos superficiales y pedregosos. Sobre la base de estas limitaciones del medio físico, se han desarrollado técnicas para superar el estrés de postplantación.

Un primer factor a considerar es la calidad del brinzal. Las condiciones de cultivo en vivero de los plantones deben estar adaptadas en todos los componentes del proceso a las características de la especie. Por ejemplo, las encinas desarrollan una raíz pivotante poco ramificada, con una mayor biomasa subterránea que aérea, en contraste con el pino carrasco. En este caso se están ensayando contenedores largos para facilitar el desarrollo de la raíz pivotante en Quercus en la fase de arraigo, con el objeto de que alcance rápidamente los horizontes profundos del suelo donde la disponibilidad hídrica es mayor, especialmente en verano. Una segunda técnica se refiere a la preparación del suelo. Las técnicas de recolección de escorrentía en clima seco y semiárido aumentan el flujo de agua hacia los brinzales plantados. Los tubos protectores reducen la transpiración de las plantas (y por lo tanto la demanda hídrica) y estimulan su crecimiento en altura. Finalmente, la fertilización del suelo utilizando enmiendas orgánicas residuales favorece, a dosis moderadas, el crecimiento de los brinzales.
En territorios con alto riesgo de incendios, la restauración forestal debe contemplar una estrategia de prevención de incendios. Con este objeto, se han desarrollado una estrategia de desbroce en matorrales altamente combustibles acompañado de la aplicación de los restos en forma de mulch y la plantación simultánea de especies arbustivas y arbóreas rebrotadoras. Con estos tratamientos se consigue reducir la combustibilidad a corto y medio plazo, y aumentar la calidad ecológica y la resiliencia del monte.

\section{Bibliografía}

Abad N., Caturla R., Baeza J., Bladé C., Vieira F., Carbó E., Valdecantos A., Bonet A., Serrasolsas I., Guàrdia R., Raventós J., Alloza J.A., Escarré A., Bellot J. \& Vallejo R. (1997). Regeneración de los montes quemados. In: La restauración de la cubierta vegetal en la Comunidad Valenciana. Ed. R. Vallejo. CEAM, Valencia.

Antolín C. (1998). El suelo como recurso natural en la Comunidad Valenciana. Antolín C. coordinadora. Serie Cartografía temática. Conselleria Obras Públicas y Urbanismo.

Bautista, S., Bellot, J. \& Vallejo, V.R. (1994). Efectos de a siembra de herbáceas y la cubierta de paja sobre la escorrentía y la erosiónm post-incendio en ambiente semiárido. Geomorfología en España, J. Arnáez et al eds. 189-199.

Bautista, S., Gimeno, T., Mayor, A.G. \& Gallego, D. (2004). El tratamiento de la madera quemada tras los incendios forestales. In: Avances en el estudio de la gestión del monte mediterráneo. V.R. Vallejo \& J.A. Alloza eds. Pp. 547-570. Fundación CEAM, Valencia. 
Boix-Fayos C., Martínez-Mena M., Calvo-Cases A.. \&Castillo V. (2003). Erosion rates in Alicante and Murcia (Spain). An overview of results, methods and scales of the last two decades. In: Briefing Papers of the first SCAPE workshop in Alicante (ES). 14-16 june, 2003. 41-49 pp.

Castillo V. \& Albadalejo J. (1992). Modelos para la predicción de la herosión hídrica, estado actual y nuevas líneas de investigación. In: Ecosistemas n 3. 28-32 pp.

Cerdá, A. 1999. Parent material and vegetation affect soil erosion in eastern Spain. Soil Sci. Soc. Am. J. 63. 362-369 pp.

COPUT (1998). Litología, aprovechamiento de rocas industriales y riesgo de deslizamiento en la Comunidad Valenciana.. Cartografía temática, 5. 86 pp. CD-ROM

Esteve M, Ferrer D., Ramírez L., Calvo J. F., Suárez M. L. \& Vidal-Abarca M. R. (1990). Restauración de la vegetación en ecosistemas áridos y semiáridos: algunas reflexiones ecológicas. In: Ecología. Fuera de serie n 1. 497-510.

FAO (1979). A provisional methodology for soil degradation assessment. Rome.

Ferran A., Serrasolsas I. \& Vallejo R. (1992). Soil evolution after fire in Quercus ilex and Pinus halepensis forests. In: Talle A. \& Jeffers J. (Eds.). Responses of Forest Ecosystems to Environmental Changes. Elsevier. 397-404.

Gallegos V., Navarro R., Fernández P. \& Valle G. (2003). Postfire regeneration in Pinus pinea L. And Pinus pinaster Aiton in Andalucia (Spain). Environmental Management Vol. 31, $n^{\circ} 1$ : 86-99.

Gandullo J.M. \& Sánchez Palomares O. (1994). Estaciones ecológicas de los pinares españoles. ICONA. Colección Técnica. Madrid.

Kazanis D.\& Arianoutsou M. (2002). Long term post-fire of Pinus halepensis forest of Central Greece: plant community patterns. In: Forest Fire Research \& Wildland Fire Safety. Ed. Viegas. Millpress. Rotterdam.

Kosmas (1999). The Medalus proyect Mediterranean desertification and land use. Manual on key indicators of desertification and mapping environmentally sensitive areas to desertification. Kosmas, Kirby and Geeson. Ed. European Commission. Luxembourg.

Lepart J.\& Debussche M. (1992). Human Impact on Landscape Patterning: Mediterranean Examples. In: Hansen \& di Castri (Eds.). Landscape Boundaries. SpringerVerlag. 76-106.

MMA, 2000. Guía para la elaboración de estudios del medio físico. Contenido y metodología. Ministerio de Medio Ambiente. Madrid.

Montero de Burgos \& González Rebollar (1983). Diagramas bioclimáticos. ICONA.

Montero, J.L. \& Alcanda, P. (1993). Reforestación y biodiversidad. Montes. 33: 57-76.
Ne'eman G., Fotheringham C. \& Keely E. (1999). Patch to landscape patterns in post fire recruitment of a serotinous conifer. Plant Ecology. 145: 235-242.

Ne'eman G. \& Trabaud L. (Eds.) (2000). Ecology, biogeography and management of Pinus halepensis and Pinus brutia. Backhuys Publisers. Leiden. The Nederlands.

Pausas J. (2001); Resprouting vs seeding - a Mediterranean perspective. Oikos, 94, 1: 193-194.

Pausas J., Vallejo R. (1999). The role of fire in European Mediterranean ecosystems. In: Chuvieco E. (Ed.). Wildfires in the European Basis. Springer. Berlin. 3-16.

Pérez Cueva, A.J. (1994). Atlas climático de la Comunidad Valenciana. Colección Territori, $n^{\circ}$ 4. Conselleria Obras Públicas. Generalitat Valenciana.

Robichaud P., Beyers J. \& Neary D. (2000). Evaluating the effectiveness of postfire rehabilitation treatments. USDA Forest Service. Rocky Mountain Research Station.

Rodrigo A., Broncano M.J. \& Retana J. (1999). Regeneration patterns of Mediterranean forest communities after large wildfires: Is autosuccession the only response? In: Proceedings of the Symposium "Forest Fires: Needs and innovations" held in Athens, 18-19. November 1999. DELFI Concerted Action, CINAR-EC, DGXII. 291-294.

Ruiz De La Torre, J. (1990). Mapa forestal de España. Memoria General. ICONA.

Sánchez Díaz J. (2001). La desertificación en la Comunidad Valenciana. In: Jornadas sobre la desertificación en el marco del Plan Forestal de la Comunidad Valenciana. Conselleria de Medio Ambiente. Generalitat Valenciana.

Sánchez, J.R. (1997). Estimación de las pérdidas erosivas inducidas por las técnicas de preparación del suelo previa a la reforestación en el sur de la Comunidad Valenciana. Tesis Doctoral. Dpto. Ecología. Universidad de Alicante.

Thornes, J.B. (1995). Mediterranean desertification and the vegetation cover. In: Fantechi R., Balabanis P. \& Rubio J.L. (Eds.). Desertification in a European context. European Commission. Luxembourg. 169-194.

Trabaud, L. (1994). Postfire Plant Community Dynamics in the Mediterranean Basin. In: Moreno J.M. \& Oechel W.C. (Eds.). The role of fire in Mediterranean Type Ecosystems. 1-15.

Trabaud, L. (1999). Recuperación y regeneración de ecosistemas mediterráneos incendiados. In: Serie Geográfica. № 7, 1997/1998. Universidad de Alcalá. 37-47.

Vallejo, R. \& Alloza, J.A.. (1998). The restoration of burned lands: The case of Eastern Spain. In: J.M. Moreno (Ed.). Large forest fires. Backhuys Publ. Leiden. 91-108.

Vallejo, V.R. (Ed.) (1996). La restauración de la cubierta vegetal en la Comunidad Valenciana. CEAM. Valencia. 